\title{
Un anciano con ampollas hemorrágicas a distancia por enoxaparina
}

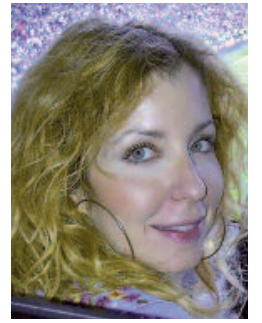

Elena González-Guerra Doctora en Medicina y Cirugía.

Médico adjunto del Servicio de Dermatología. Hospital Clínico San Carlos. Madrid.

Médico colaborador en Docencia Práctica de Dermatología.

Facultad de Medicina. Universidad Complutense. Madrid.

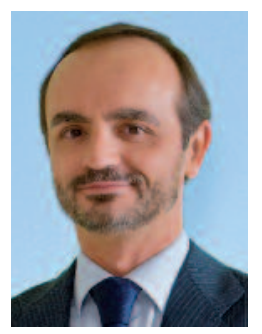

Eduardo López Bran Profesor titular de Dermatología. Departamento de Medicina.

Facultad de Medicina. Universidad Complutense. Madrid.

Jefe del Servicio de Dermatología.

Hospital Clínico San Carlos. Madrid.
Mi paciente es un anciano de 86 años con antecedentes de hipertensión arterial, dislipidemia, hiperplasia benigna de próstata, fibrilación auricular, faquectomía bilateral y cirugía de hernia discal. Como suele ocurrir en edades avanzadas, se encuentra polimedicado de forma habitual con atenolol, acenocumarol, atorvastatina y dutasterida/tamsulosina. Tres años antes de ser visto en consulta de dermatología fue diagnosticado de un carcinoma renal de células claras con áreas de diferenciación papilar, por lo que se le practicó una nefrectomía derecha, desarrollando a continuación insuficiencia renal crónica. En el estudio de extensión se objetivaron metástasis pulmonares. Comenzó tratamiento para el cáncer con pazopanib (fármaco inhibidor de la proteína cinasa). Tras una de sus visitas al Servicio de Oncología y sin previo diagnóstico de accidente trombótico, se le modifica el tratamiento anticoagulante cambiando acenocumarol por enoxaparina subcutánea en dosis de $40 \mathrm{mg} / 12 \mathrm{~h}$. La enoxaparina es la sal sódica de una heparina de bajo peso molecular. Está indicada como profilaxis y tratamiento de trastornos tromboembólicos.

Tras una semana de tratamiento, el paciente fue derivado a nuestra consulta por la presencia de tres ampollas de contenido hemático, tensas, de unos $5 \mathrm{~mm}$, localizadas en el dor-

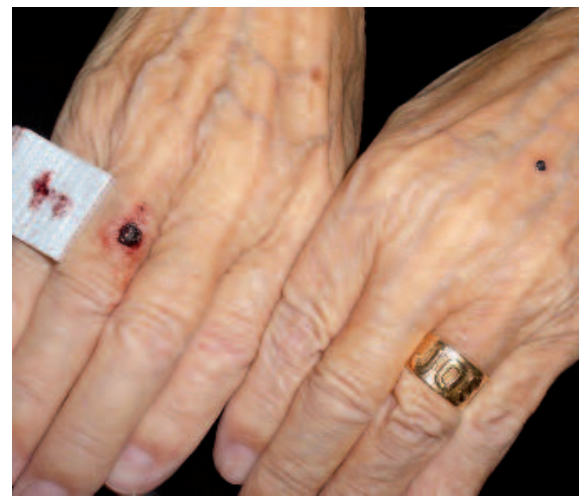

Figura 1. Ampollas hemorrágicas, tensas, localizadas en el dorso de las manos de un paciente tratado con enoxaparina.

so de ambas manos y muslo izquierdo (fig. 1).

Se realizó analítica con recuento de plaquetas y estudio de coagulación (tiempo de protrombina, actividad de protrombina, índice internacional normalizado, tiempo de tromboplastina y fibrinógeno), que fueron normales. De forma simultánea, se practicó una biopsia de una de las lesiones donde se observaba, en la dermis superior, amplias áreas hemorrágicas con algunos neutrófilos de predominio perivascular sin que se apreciase afectación de la pared vascular (fig. 2).

Con estos datos, establecimos el diagnóstico de dermatosis ampollosa hemorrágica a distancia por enoxaparina.

Tras 3 semanas, las lesiones cutáneas se resolvieron sin secuelas, sin 


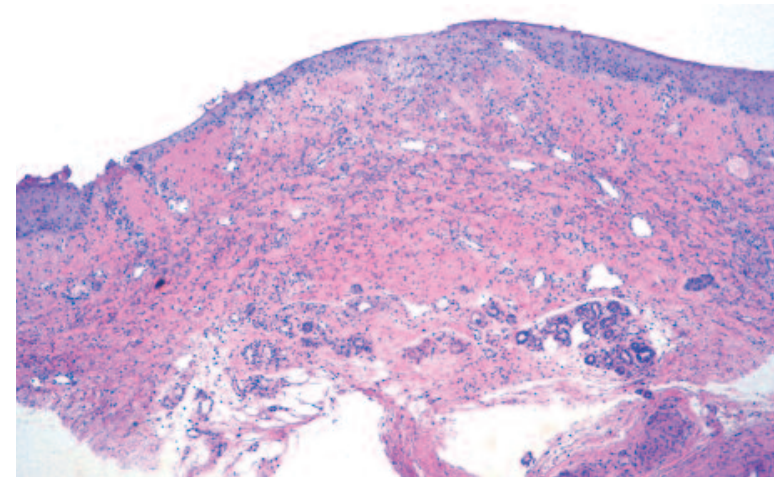

Figura 2. En la biopsia se observa la presencia de amplias áreas hemorrágicas con infiltrado neutrofílico de predominio perivascular.

modificación del tratamiento anticoagulante, ni la administración de medicación específica para ellas.

Entre las principales reacciones adversas cutáneas de la enoxaparina destacan: dolor, equimosis, necrosis cutánea, urticaria, angioedema y eccema. En 2006 se describió una nueva reacción cutánea asociada a la administración de heparina que consistía en la aparición de ampollas hemorrágicas localizadas a distancia del sitio de la inyección. Hasta la actualidad, hemos encontrado 14 casos descritos de dermatosis ampollosa hemorrágica a distancia por heparina. La causa continua siendo desconocida.

La enoxaparina sódica se caracteriza por tener una acción inhibidora del factor Xa de la cascada de la coagulación y una débil actividad antifactor IIa o antitrombínica. La combinación de estos dos mecanismos permite su uso en la profilaxis de enfermedades trombóticas, con bajo riesgo de producir trombocitopenia, a diferencia de la heparina estándar, lo que hace que no sea precisa la monitorización de los parámetros de la coagulación. La eliminación de la enoxaparina se realiza por vía renal y biliar. En los pacientes con insuficiencia renal, como en nuestro caso, es necesario un ajuste de la dosis.

Además, nuestro paciente padecía también un cáncer renal. Los accidentes tromboembólicos en las personas con cáncer son frecuentes y pro- ducen una alta morbimortalidad. De hecho, es la segunda causa de muerte en estos enfermos. El tratamiento anticoagulante se ha mostrado efectivo en estos casos ${ }^{1}$. Además, la insuficiencia renal está presente en el 50-60\% de los pacientes con cáncer, como sucedió en nuestro caso. Por ello, tanto el riesgo de tromboembolia como la presencia de insuficiencia renal son dos circunstancias a tener en cuenta a la hora de instaurar una terapia anticoagulante en pacientes con cáncer, ya sea como profilaxis o como tratamiento, como sucedió en nuestro caso por tener de forma simultánea una fibrilación auricular.

Sin embargo, en los pacientes con cáncer, el beneficio del tratamiento con heparina podría ir más allá de la prevención secundaria de la enfermedad tromboembólica. Las células tumorales producen el factor tisular y otros procoagulantes. Los tumores interactúan con el endotelio, los leucocitos, las plaquetas durante las fases de crecimiento, diseminación, y establecimiento de metástasis. Inhibir el sistema hemostático podría alterar la biología del cáncer y mejorar la supervivencia, independientemente de que exista un accidente tromboembólico o no. Un metaanálisis demostró que en pacientes con cáncer tratados con heparina de bajo peso molecular y sin trombosis se reducía un $17 \%$ la mortalidad ${ }^{2}$. Por esta razón, se cambió el tratamiento anticoagulante del paciente de acenocumarol a enoxaparina, a raíz del cual desarrolló la dermatosis ampollosa hemorrágica descrita en el caso clínico.

En el año 2006, Perrinaud et al. ${ }^{3}$ describieron por primera vez tres casos de lesiones ampollosas hemorrágicas localizadas a distancia de la zona de aplicación de la heparina (un caso con heparina clásica y dos con heparinas de bajo peso molecular). Posteriormente, se han descrito nuevos casos con el uso de heparinas de bajo peso molecular como enoxaparina, tinzaparina y bemipari$\mathrm{na}^{4}$. Las lesiones cutáneas estaban localizadas con más frecuencia en extremidades superiores, fundamentalmente en el dorso de las manos, y extremidades inferiores. En cinco de los casos publi- 
cados existía como antecedente personal la presencia de neoplasias malignas como sucedía con nuestro paciente.

El estudio histopatológico, como en el caso clínico descrito, es inespecífico: se describen vesículas y ampollas intraepidérmicas o subcórneas, de contenido hemático, sin vasculitis ni trombosis en los capilares.

En todos los casos se administraron altas dosis de heparina, por lo que una de las hipótesis planteadas en la literatura científica es que se trate de un efecto dependiente de la dosis ${ }^{5}$.

Las lesiones se resolvieron en pocos días, en la mayoría de los casos, sin necesidad de suspensión del tratamiento anticoagulante con heparina. Este hecho hace pensar que se trata de un fenómeno infradiagnosticado o poco referido en la literatura, en el que hay que pensar en los pacientes con esta clínica, aunque sea pasajera.

\section{BIBLIOGRAFÍA}

1. Cesarman-Maus G, Meillón L, Volkow P, Vargas-Ruiz AG, Cornejo P, López-Navarro O, et al. Treatment of cancer and thrombosis: practical approach. Rev Invest Clin. 2013;65(2): 174-82.

2. Scotté F, Rey JB, Launay-Vacher V. Thrombosis, cancer and renal insufficiency: low molecular weight heparin at the crossroads. Support Care Cancer. 2012;20(12):3033-42.

3. Perrinaud A, Jacobi D, Machet MC, Grodet C, Gruel Y, Machet Bullous L. Hemorrhagic dermatosis occurring at sites distant from subcutaneous injections of heparin: three cases. J Am Acad Dermatol. 2006;S5-7.

4. Villanueva CA, Nájera L, Espinosa P, Borbujo J. Bullous hemorrhagic dermatosis at distant sites: a report of 2 new cases due to enoxaparin injection and a review of the literature. Actas Dermosifiliogr. 2012;103(9):816-9.

5. Maldonado Cid P, Moreno Alonso de Celada R, Herranz Pinto P, Noguera Morel L, Feltes Ochoa R, Beato Merino MJ, et al. Bullous hemorrhagic dermatosis at sites distant from subcutaneous injections of heparin: A report of 5 cases. J Am Acad Dermatol. 2012;67:220-2. 